\title{
Dipyrone Increases the Blood Flow of Arterial Dorsal Skin Flaps: Are Prospective Studies Always Properly Designed?
}

\author{
Luca La Colla $\cdot$ Alberto Mangano • \\ Alessandro Mangano $\cdot$ Andrea Albertin
}

Published online: 26 February 2009

(c) Springer Science+Business Media, LLC and International Society of Aesthetic Plastic Surgery 2009

We read with interest the article published by Gulmez et al. [1]. While the authors are to be commended for their goal of conducting a prospective, randomized, double-blind trial about the effect of dipyrone on blood flow and necrosis (i.e., flap survival), there are several issues with respect to the design of the study and the statistical analysis used. With respect to study design, the major issue is that there is no reference to any sample size calculation. In fact, in order for a particular finding to be claimed as significant (or not), the study must have enough power. In this particular case, the expected change in blood flow should be stated and from that the sample size should be calculated (e.g., considering the "standard" $\alpha$ error of 0.05 and a power of 0.8 ). Moreover, there is no reference to the randomization method.

Another critical point we want to bring up is the statistical analysis used. The authors state that "Student's $t$ test at a $p$ level of significance less than 0.05 was used to compare the percentage of necrotic area and blood flow changes from baseline for each time point measure." This

L. La Colla $(\bowtie)$

Department of Anesthesiology, Vita-Salute San Raffaele

University School of Medicine, IRCCS H San Raffaele,

Via Olgettina 60, Milan 20132, Italy

e-mail:1.1acolla@studenti.hsr.it

\section{A. Mangano}

Vita-Salute San Raffaele University School of Medicine, IRCCS H San Raffaele, Milan, Italy

\section{A. Mangano}

University of Milan Dental School, Milan, Italy

\section{A. Albertin}

Department of Anesthesiology, IRCCS Multimedica, Sesto San Giovanni, Milan, Italy may not be the correct way to go. Are all the variables normally distributed? If not, nonparametric tests should be used. Moreover, this study involves both tests between groups and tests within the same individuals (i.e., repeated measurements on blood flow and fraction of necrosis). Therefore, close attention should be paid to whether use of a $t$ test for unpaired or paired data is appropriate and, similarly, to the use of either a Wilcoxon rank-sum test or signed-rank test. While this study is extremely interesting for plastic surgeons, appropriate statistical analysis of data from trials is crucial for adequate conclusions.

In addition, we have recently shown that the anesthetic technique used affects regional blood flow in spine surgery [2]. Because most patients undergoing plastic surgery procedures are under general anesthesia, it would be interesting to investigate the effect of different anesthetic techniques on flap vascularization and viability.

We hope our suggestions will be useful to other authors who will be involved in similar studies in the future.

\section{References}

1. Gulmez SE, Serel S, Uluc A, Can Z, Ergün H (2008) Dipyrone increases the blood flow of arterial dorsal skin flaps. Aesthetic Plast Surg 32:766-770

2. Albertin A, La Colla L, Gandolfi A, Colnaghi E, Mandelli D, Gioia G, Fraschini G (2008) Greater peripheral blood flow but less bleeding with propofol versus sevoflurane during spine surgery: a possible physiologic model? Spine 33:2017-2022 